EDITORIAL

\title{
A Rare Case of Pseudotumoral Ureteric Tuberculosis Causing Forniceal Rupture
}

MOHAMED HAJRI ${ }^{1}$, HOUSSEM BEN HADJ ALOUANE ${ }^{2}$ WAEL FERJAOUI ${ }^{1 *}$, SEIFEDDINE BACCOUCHE ${ }^{1}$, LASAAD GHARBI $^{1}$, HAFEDH MESTIRI ${ }^{1}$, RACHED BAYAR ${ }^{1}$, DHOUHABACHA ${ }^{3}$, SANA BEN SLAMA ${ }^{3}$, AHLEM LAHMER ${ }^{3}$

${ }^{1}$ Department of General Surgery, Mongi Slim University Hospital, Faculty of Medicine of Tunis, University of Tunis el Manar

${ }^{2}$ Department of Urology, La Rabta University Hospital, Tunis, Tunisia

${ }^{3}$ Department of Pathology, Mongi Slim University Hospital, Faculty of Medicine of Tunis, University of Tunis el Manar

*Correspondence to Wael Ferjaoui Tel: +216 52430099/ E mail: farjaouiwael4@gmail.com

https://orcid.org/0000-0002-4279-9370

A 55-year-old woman, with no medical history, presented with acute right flank pain. She had no history of other urinary complaints. On physical examination, the patient was tachycardic (pulse rate: $100 \mathrm{bpm}$ ) and tachypneic (respiratory rate: 24 breaths $/ \mathrm{min}$ ), blood pressure was $11 / 6$ and temperature was $37.4^{\circ}$.

The abdominal examination showed severe tenderness in the right flank and the right iliac fossa. All blood reports were normal, including C-reactive protein, cell blood count and serum creatinine. Computed Tomography of the abdomen revealed a right hydronephrosis with delayed phase contrast leak and a retroperitoneal mass of $48 \times 36 \mathrm{~mm}$ of unknown nature, enhanced after contrast injection, which seemed to compress the right ureter causing the forniceal rupture.

A double $\mathrm{J}$ ureteral stent was insterted into the right renal cavities with favorable evolution and immediate resolution of pain. Surgical management of the mass was scheduled one month later after the inflammatory phase and resorption of the urinoma. The patient underwent an exploratory laparotomy. Intraoperatively, a tissular retroperitoneal mass of $4 \mathrm{~cm}$ was discovered which invadedthe right proximal ureter as well asthe duodenum and the ileocecal pedicle (Figure 1). Resection of the tumor was performed as well as a segmental ureterectomy, right colectomy, and resection of a small portion of the duodenum. Both ureteric and colic anastomosis were then performed along with duodenal suture.

The post operative course was uneventful.

Histology showed epithelial giant cells with the presence of caseous necrosis in the ureteric wall, which confirmed the diagnosis of tuberculosis (Figure 2).

Anti-tuberculosis therapy was started. The patient is currently doing well, four months after his operation.

Tuberculosis is still endemic in Tunisia. The genitourinary form accounts for 8 to $20 \%$ of extrapulmonary tuberculosis cases $^{1}$. Pseudotumoral involvement of ureter by tuberculosis is uncommon. It is probably due to an extending fibro-inflammatory process in the thickening of the ureteral wall ${ }^{2,3}$.

A similar case has recently been published by Dhangar et $\mathrm{al}^{2}$. But the specificity of our case is that the ureteral stenosis due to pseudotumoral mass was responsible for an upstream forniceal rupture. This observation clarify the need for clinicians to consider a ureteral tuberculosis diagnosis whenever ureteral thickening is revealed especially in endemic countries. That's why more means of diagnosis such as Quantiferon test and polymerase chain reaction (PCR) should be used before proceeding to unsuited major surgery ${ }^{1}$.

Figure 1: Intraoperative aspect of the ureteric mass invading the duodenum and the ileocecal pedicle.

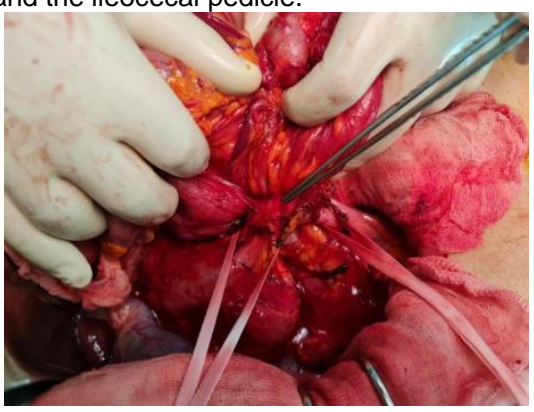

Figure 2: Histologic aspect of the ureteric mass showing epithelial giant cells with caseous necrosis.
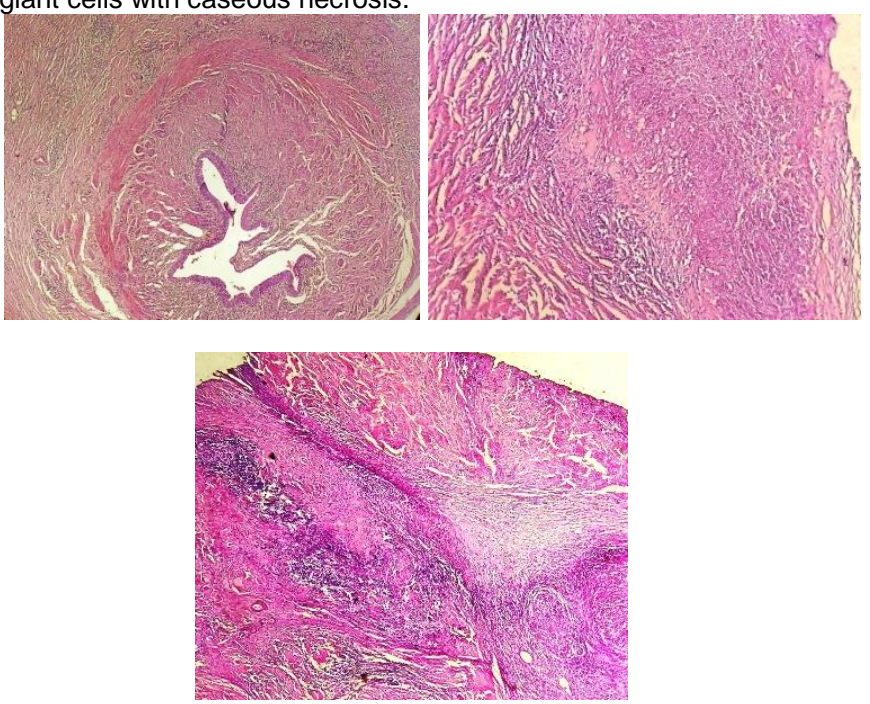

\section{REFERENCES}

1. Lahouar R, Khalifa BB, Baba NE, Gazzah W, Naouar S, Braiek S, et al. Unusual presentations of urogenital tuberculosis. Int J Surg Case Rep. Elsevier; 2020;77:769- 72.

2. Dhangar SP, Syed AA, Khadatkar A, Shengal M. A rare case of isolated lower ureteric tuberculosis mimicking a ureteric tumor.

3. Bouchikhi A-A, Amiroune D, Tazi MF, Mellas S, Elammari JE, El Fassi MJ, et al. Pseudotumoral tuberculous ureteritis: a case report. J Med Case Reports. BioMed Central; 2013;7(1):1-3. 